\title{
Polymorphisms in the endothelial nitric oxide synthase gene associated with recurrent miscarriage
}

\author{
L. Luo ${ }^{1,2}$, D.H. Li ${ }^{2}$, S.G. Wei ${ }^{1}$, H.B. Zhang ${ }^{1}$, S.B. Li ${ }^{1}$ and J. Zhao ${ }^{2}$ \\ ${ }^{1}$ Department of Forensic Science, School of Medicine, \\ Xi' an Jiaotong University/Key Laboratory of Ministry of Public Health for \\ Forensic Science, Xi'an, Shaanxi, China \\ ${ }^{2}$ Reproductive Medical Center of the Fourth Hospital of Xi'an, \\ Xi'an, Shaanxi, China \\ Corresponding authors: S.B. Li / J. Zhao \\ E-mail: shbinlee@mail.xjtu.edu.cn / zhaojingfck@163.com
}

Genet. Mol. Res. 12 (3): 3879-3886 (2013)

Received January 29, 2013

Accepted July 20, 2013

Published September 23, 2013

DOI http://dx.doi.org/10.4238/2013.September.23.6

\begin{abstract}
Endothelial nitric oxide synthase (eNOS) is an enzyme that influences placental human chorionic gonadotropin production during gestation. Previous studies have indicated an association between eNOS activity, implantation, and maintenance of pregnancy, but proposed associations between polymorphisms of the eNOS gene and recurrent miscarriage (RM) are controversial. To identify markers contributing to the genetic susceptibility to RM, we examined the potential association between RM and 8 single nucleotide polymorphisms (SNPs; rs1799983, rs2070744, rs11771443, rs3918188, rs2853796, rs7830, rs1541861, and rs2853792) of the eNOS gene using the MassARRAY system (Sequenom, USA). The enrolled participants included $192 \mathrm{RM}$ patients and 201 women with normal fertility as controls. The results showed that rs1799983 at exon 7 of the eNOS gene was significantly associated with RM (genotype: chi-square $=15.071, \mathrm{P}=0.001$; allele: chi-square $=6.250, \mathrm{P}=0.016$ ). Another significant association was observed for rs11771443 in the
\end{abstract}


promoter (genotype: chi-square $=6.259, \mathrm{P}=0.044$; allele: chi-square $=7.076, \mathrm{P}=0.008)$. Furthermore, strong linkage disequilibrium was observed in 3 blocks ( $\mathrm{D}^{\prime}>0.9$ ), and significantly fewer T-T-G haplotypes (chi-square $=5.981, \mathrm{P}=0.015$ ) residing in block 1 were found in $\mathrm{RM}$ patients. These findings point to a role for eNOS gene polymorphisms in RM in the Chinese Han population and may be informative for future genetic or neurobiological studies of RM.

Key words: Endothelial nitric oxide synthase; Recurrent miscarriage; Single-nucleotide polymorphisms

\section{INTRODUCTION}

Approximately 15 to $20 \%$ of clinically recognized pregnancies end in miscarriage, and approximately 1 to $5 \%$ of pregnant women experience recurrent miscarriage (RM), of which 40 to $55 \%$ are induced by unknown causes (Yamada et al., 2001). Although many etiological factors causing RM are known, such as parental chromosome abnormalities, uterine abnormalities, hereditary thrombophilias, endocrinologic disorders, immunologic factors, infections, and nutritional and environmental factors ( $\mathrm{Li}$ et al., 2002), most RM cases remain idiopathic.

Endothelial nitric oxide synthase (eNOS), which oxidizes its substrate L-arginine to L-citrulline, is the main enzyme responsible for the production of vascular nitric oxide (NO) (Moncada and Higgs, 1993). During the first trimester, eNOS is expressed in the terminal chorionic villous vessels and in the cyto- and syncytiotrophoblast layers. The level of eNOS increases as gestation progresses and is significantly enhanced in certain pathological conditions (Ayuk et al., 2002). Furthermore, the placental production of human chorionic gonadotropin, an important angiogenic factor for neoangiogenic interaction and trophoblast migration (Zygmunt et al., 2002), is modulated by eNOS during gestation (Sanyal et al., 2000). Abnormal levels of NO have been reported in the placentae from preeclamptic pregnancies as well as from term pregnancies with fetal growth retardation (Myatt et al., 1997). In mice, placental NO production mediates abortion induced by lipopolysaccharide (Haddad et al., 1995), whereas pharmacological inhibition of NO release with aminoguanidine successfully rescues lipopolysaccharide-induced abortion (Athanassakis et al., 1999). Thus, eNOS and NO may contribute to pregnancy maintenance by controlling both endocrine function and vascular tone.

Two single-nucleotide polymorphisms (SNPs) associated with eNOS protein expression, including rs1799983 (894G > T, Glu298Asp) in exon 7 and rs2070744 (-786T $>$ C) in the promoter region have been well described in the eNOS gene (Nakayama et al., 1999; Hefler et al., 2002). The rs1799983 SNP is associated with various vascular diseases related to pregnancy (Yoshimura et al., 2001; Hefler et al., 2002; Hillermann et al., 2005; Huber et al., 2005; Suryanarayana et al., 2006). A systematic review has indicated that rs1799983 may be correlated with recurrent spontaneous abortion (Su et al., 2011). The rs2070744 SNP has been observed in patients with vascular disease (Khurana et al., 2003; Rossi et al., 2003; Guang-Da et al., 2005); it inhibits the promoter activity of eNOS. However, whether rs2070744 is associated with RM remains controversial (Serrano et al., 2004; Shin et al., 2010). In addition to reports of these variants, a limited number of studies have investigated a possible association between RM and other polymorphisms of the eNOS gene. Thus, a larger number of patients must be assessed for conclusive results.

In the present study, we investigated the crucial and controversial role of the eNOS polymorphisms in RM. We systematically identified 8 genetic polymorphisms in eNOS and 
conducted a case-control association analysis of the variants and their haplotypes to determine the susceptibility of variants and haplotypes to RM.

\section{MATERIALAND METHODS}

\section{Subjects}

The study group consisted of 340 women (age range, 22-43 years; mean age $\pm \mathrm{SD}, 30.56$ \pm 4.58 years) who were diagnosed with at least 3 consecutive spontaneous abortions; these patients were enrolled in the study at the Fourth Hospital of Xi'an (Xi'an, China). All RM patients underwent careful clinical examinations, including hysteroscopy or hysterosalpingography, transvaginal ultrasound, serial endometrial biopsies, and analyses of tissue antibodies and autoantibodies. The results of the clinical examinations showed that they had no identifiable causes for their miscarriages, including chromosomal abnormalities or anatomic, endocrine, cytogenetic, or autoimmune causes, and antibody screens for toxoplasmosis, cytomegalovirus, rubella, human immunodeficiency virus, group B streptococci, chlamydia trachomatis, and hepatitis B and C were negative. Age- and ethnicity-matched subjects included 115 women (age range, 21-44 years; mean age \pm $\mathrm{SD}, 31.02 \pm 4.36$ years), each of whom had a history of at least 1 live birth and no pregnancy loss, were recruited as healthy controls at the Fourth Hospital of Xi'an. The following exclusion criteria were used: taking prescribed medications that could affect the central nervous system; a history of seizures, hematological disease, or severe liver or kidney damage; smoking; hypertension; and previous use of oral contraceptives. This study was approved by the ethics committee of the Fourth Hospital of Xi'an, and written informed consent was obtained from all participants.

\section{SNP selection}

The genomic length of eNOS is $21 \mathrm{~kb}$. SNPs in the promoter region, untranslated region, exons, and introns of the eNOS gene were systematically screened. Marker selection was based on the following criteria. First, we examined tagSNPs in Haploview version 4.2 (Daly Lab Inc., Cambridge, MA, USA) using the Han Chinese in Beijing population and a minor allele frequency cut-off of below 5\% (HapMap Data Release 27). Second, the linkage disequilibrium (LD) pattern of the gene was determined in the Chinese population using the preliminary data from HapMap. Third, 8 SNPs were selected for genotyping. Previous studies have shown significant associations between SNPs and RM. The rs1799983 SNP is located in exon 7, rs2070744 and rs 11771443 in the promoter, rs1541861 in intron 8, rs3918188 and rs2853792 in intron 12, rs2853796 in intron 13, and rs7830 in intron 22. These SNPs were further analyzed in an association study.

\section{Genotyping}

Genomic DNA was extracted from blood leukocytes using an EZNATM Blood DNA Midi Kit (Omega Bio-Tek, Norcross, GA, USA) according to the manufacturer protocol. Genotyping for 8 SNPs was performed using the MassARRAY platform (Sequenom, San Diego, CA, USA) by means of a matrix-assisted laser desorption ionization-time of flight mass spectrometry method according to manufacturer instructions. Primers were designed using Sequenom software (Table 1). 
Table 1. Primer sequences used for genotyping eNOS gene SNPs with the MALDI-TOF Sequenom platform.

\begin{tabular}{llll}
\hline SNPs & Forward primers & Reverse primers & Extension primers \\
\hline rs1799983 & ACGTTGGATGAAACGGTCGCTTCGACGTG & ACGTTGGATGACCTCAAGGACCAGCTCGG & TGCAGGCCCCAGATGAG \\
rs2070744 & ACGTTGGATGACTGTAGTTTCCCTAGTCCC & ACGTTGGATGAGGTCAGCAGAGAGACTAGG & CAAGCTCTTCCCTGGCC \\
rs11771443 & ACGTTGGATGCGTCTGTGGGCGTAACATC & ACGTTGGATGGAAGGATCAGGCCCACAATG & CCGCTGGGCTGATGTA \\
rs3918188 & ACGTTGGATGAAAAGTGGGAGCAAGGCACA & ACGTTGGATGACTTCACTGAGACTGAAGGG & AGGCACACGTACAAGGGC \\
rs2853796 & ACGTTGGATGAAGACAAGGTTTCACAGGG & ACGTTGGATGAGAGGCAGAGACCCTGAAG & ACAGGCTAGGCCCAGC \\
rs7830 & ACGTTGGATGCTGTCCCTAGATTGTGTGAC & ACGTTGGATGCGGCTGCATGACATTGAGAG & CCTTCAGGCAGTCCTTTAGTCC \\
rs1541861 & ACGTTGGATGATCAGATTCTCAAAGGGGAC & ACGTTGGATGACCTCAGAGCAGAGAGGGA & ATTCTCAAAGGGGACCTTGACTCGGC \\
rs2853792 & ACGTTGGATGCAAATAAAATAAAATGCTGG & ACGTTGGATGGTTTGTAAAAATCCACAGAGC & AATGCTGGATAGTGTTGC \\
\hline
\end{tabular}

\section{Statistical analysis}

Each SNP was tested for deviation from Hardy-Weinberg equilibrium using Pearson's chi-square test or Fisher's exact test. The association between genetic polymorphisms was examined using the chi-square test and Fisher's exact test. The odds ratios (ORs) and 95\% confidence intervals $(95 \% \mathrm{CIs})$ were calculated to estimate the strength of association through unconditional logistic regression analysis. The common genotype/allele served as a reference for calculating OR. All statistical tests were conducted in SPSS 16.0 for Windows (SPSS Inc., Chicago, IL, USA). Differences were considered significant if the P value was smaller than 0.05 . For haplotype analyses, the $\mathrm{P}$ value was based on a comparison of the frequency distribution of all possible combinations of haplotypes that contained a certain SNP that was significantly associated with RM. Pairwise LD statistics (D' and $\mathrm{r}^{2}$ ) and haplotype frequencies were computed using Haploview 4.0 to construct haplotype blocks. Haplotypes with a frequency of less than 5\% were combined with the rare haplotype group. The frequency of all haplotypes was compared between case and control participants using Pearson's chi-square test.

\section{RESULTS}

The distribution frequencies of the 8 genotyped SNPs were in agreement with HardyWeinberg equilibrium. LD analyses of RM patients and healthy controls revealed that 3 SNPs (rs11771443, rs2070744, rs1799983), 2 SNPs (rs1541861, rs2853792), 2 SNPs (rs3918188, rs2853796) were located in haplotype blocks 1, 2, and 3, respectively ( $\mathrm{D}^{\prime}>0.9$; Figure 1).

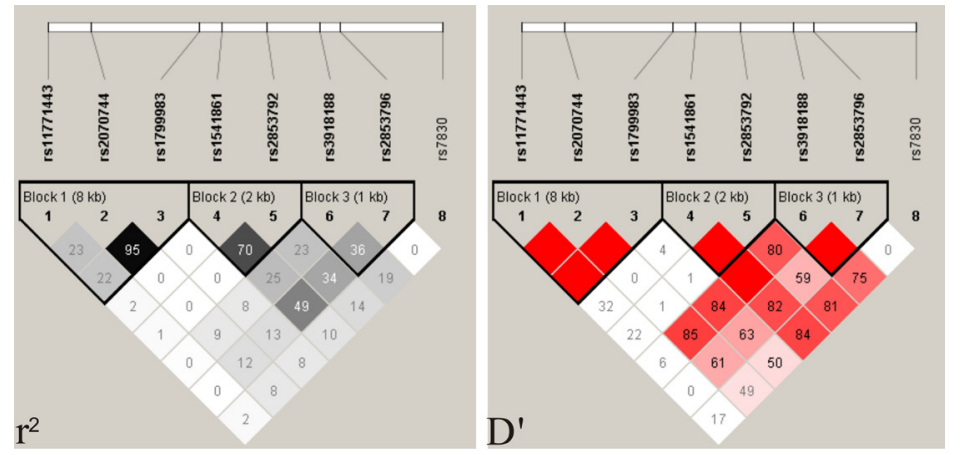

Figure 1. Linkage disequilibrium (LD) plot of 8 single nucleotide polymorphisms (SNPs) in the endothelial nitric oxide synthase gene. Values in squares are the pairwise calculation of $\mathrm{r}^{2}$ (left) or D' (right). Black squares indicate $\mathrm{r}^{2}=$ 1 (i.e., perfect LD between a pair of SNPs). Empty squares indicate D' = 1 (i.e., complete LD between a pair of SNPs). 
The genotype distribution, allelic frequencies, and haplotypes in RM patients and healthy controls along with the results of statistical analysis are shown in Tables 2 and 3.

\begin{tabular}{|c|c|c|c|c|}
\hline Identity & Genotype/allele & Controls [N (\%)] & Case $[\mathrm{N}(\%)]$ & OR, 95\%CI \\
\hline \multirow[t]{7}{*}{ rs1799983 } & $\mathrm{G} / \mathrm{G}$ & $154(76.617)^{\#}$ & $118(61.458)$ & 1.0 (reference) \\
\hline & $\mathrm{G} / \mathrm{T}$ & $41(20.398)$ & $72(37.500)$ & $2.292,1.458-3.603$ \\
\hline & $\mathrm{T} / \mathrm{T}$ & $6(2.985)$ & $2(1.042)$ & $0.435,0.086-2.194$ \\
\hline & $\chi^{2}, \mathrm{P}$ & $15.071,0.001^{*}$ & & \\
\hline & $\mathrm{G}$ & 349 (86.816) & $308(80.208)$ & $1.625,1.108-2.383$ \\
\hline & $\mathrm{T}$ & $53(13.184)$ & 76 (19.792) & \\
\hline & $\chi^{2}, \mathrm{P}$ & $6.250,0.016^{*}$ & & \\
\hline \multirow[t]{7}{*}{ rs2070744 } & $\mathrm{T} / \mathrm{T}$ & 153 (76.119) & $128(66.667)^{\#}$ & 1.0 (reference) \\
\hline & $\mathrm{T} / \mathrm{C}$ & $41(20.398)$ & $55(28.646)$ & $1.603,1.005-2.559$ \\
\hline & $\mathrm{C} / \mathrm{C}$ & $7(3.483)$ & $9(4.688)$ & $1.537,0.557-4.242$ \\
\hline & $\chi^{2}, \mathrm{P}$ & $4.312,0.116$ & & \\
\hline & $\mathrm{T}$ & $347(86.318)$ & $311(80.990)$ & $1.481,1.011-2.170$ \\
\hline & $\mathrm{C}$ & $55(13.682)$ & $73(19.010)$ & \\
\hline & $\chi^{2}, \mathrm{P}$ & $4.091,0.053$ & & \\
\hline \multirow[t]{7}{*}{ rs11771443 } & $\mathrm{T} / \mathrm{T}$ & $78(38.806)$ & $57(29.688)^{\#}$ & 1.0 (reference) \\
\hline & $\mathrm{T} / \mathrm{C}$ & $84(41.791)$ & $79(41.146)$ & $1.287,0.813-2.037$ \\
\hline & $\mathrm{C} / \mathrm{C}$ & $39(19.403)$ & $56(29.167)$ & $1.965,1.153-3.347$ \\
\hline & $\chi^{2}, \mathrm{P}$ & $6.259,0.044 *$ & & \\
\hline & $\mathrm{T}$ & $240(59.701)$ & $193(50.260)$ & $1.497,1.128-1.986$ \\
\hline & $\mathrm{C}$ & $162(40.299)$ & $191(49.740)$ & \\
\hline & $\chi^{2}, \mathrm{P}$ & $7.076,0.008^{*}$ & & \\
\hline \multirow[t]{7}{*}{ rs3918188 } & $\mathrm{C} / \mathrm{C}$ & $69(34.328)$ & $62(32.292)^{\#}$ & 1.0 (reference) \\
\hline & $\mathrm{C} / \mathrm{A}$ & $89(44.279)$ & $97(50.521)$ & $1.213,0.775-1.898$ \\
\hline & $\mathrm{A} / \mathrm{A}$ & $43(21.393)$ & $33(17.188)$ & $0.854,0.484-1.508$ \\
\hline & $\chi^{2}, \mathrm{P}$ & $1.829,0.401$ & & \\
\hline & $\mathrm{C}$ & $227(56.468)$ & $221(57.552)$ & $0.957,0.721-1.269$ \\
\hline & $\mathrm{A}$ & $175(43.532)$ & $163(42.448)$ & \\
\hline & $\chi^{2}, \mathrm{P}$ & $0.094,0.773$ & & \\
\hline \multirow[t]{7}{*}{ rs2853796 } & $\mathrm{T} / \mathrm{T}$ & $101(50.249)$ & $91(47.396)^{\#}$ & 1.0 (reference) \\
\hline & $\mathrm{T} / \mathrm{G}$ & $72(35.821)$ & $74(38.542)$ & $1.141,0.742-1.755$ \\
\hline & $\mathrm{G} / \mathrm{G}$ & $28(13.930)$ & $27(14.063)$ & $1.070,0.588-1.950$ \\
\hline & $\chi^{2}, \mathrm{P}$ & $0.360,0.835$ & & \\
\hline & $\mathrm{T}$ & $274(68.159)$ & $256(66.667)$ & $1.070,0.794-1.442$ \\
\hline & $\mathrm{G}$ & $128(31.841)$ & $128(33.333)$ & \\
\hline & $\chi^{2}, \mathrm{P}$ & $0.199,0.704$ & & \\
\hline \multirow[t]{7}{*}{ rs 7830} & $\mathrm{C} / \mathrm{C}$ & 95 (47.264) & $83(43.229)$ & 1.0 (reference) \\
\hline & $\mathrm{C} / \mathrm{A}$ & $88(43.781)$ & $89(46.354)$ & $1.158,0.763-1.756$ \\
\hline & $\mathrm{A} / \mathrm{A}$ & $18(8.955)$ & $20(10.417)$ & $1.272,0.631-2.565$ \\
\hline & $\chi^{2}, \mathrm{P}$ & $0.714,0.700$ & & \\
\hline & $\mathrm{C}$ & $278(69.154)$ & $255(66.406)$ & $1.134,0.841-1.530$ \\
\hline & $\mathrm{A}$ & $124(30.846)$ & $129(33.594)$ & \\
\hline & $\chi^{2}, \mathrm{P}$ & $0.679,0.445$ & & \\
\hline \multirow[t]{7}{*}{ rs1541861 } & $\mathrm{A} / \mathrm{A}$ & $119(59.204)$ & $120(62.500)$ & 1.0 (reference) \\
\hline & $\mathrm{A} / \mathrm{C}$ & $63(31.343)$ & $55(28.646)$ & $0.866,0.557-1.347$ \\
\hline & $\mathrm{C} / \mathrm{C}$ & $19(9.453)$ & $17(8.854)$ & $0.887,0.440-1.790$ \\
\hline & $\chi^{2}, \mathrm{P}$ & $0.452,0.798$ & & \\
\hline & $\mathrm{A}$ & $301(74.876)$ & $295(76.823)$ & $0.899,0.648-1.247$ \\
\hline & $\mathrm{C}$ & $101(25,124)$ & 89 (23.177) & \\
\hline & $\chi^{2}, \mathrm{P}$ & $0.406,0.560$ & & \\
\hline \multirow[t]{7}{*}{ rs2853792 } & $\mathrm{A} / \mathrm{A}$ & 93 (46.269) & $93(48.438)^{\#}$ & 1.0 (reference) \\
\hline & $\mathrm{A} / \mathrm{G}$ & $86(42.786)$ & $80(41.667)$ & $0.930,0.612-1.414$ \\
\hline & $\mathrm{G} / \mathrm{G}$ & $22(10.945)$ & $19(9.896)$ & $0.864,0.438-1.701$ \\
\hline & $\chi^{2}, \mathrm{P}$ & $0.230,0.891$ & & \\
\hline & $\mathrm{A}$ & $272(67.662)$ & $266(69.271)$ & $0.928,0.687-1.254$ \\
\hline & G & $130(32.338)$ & $118(30.729)$ & \\
\hline & $\chi^{2}, \mathrm{P}$ & $0.235,0.646$ & & \\
\hline
\end{tabular}

*Significant results $(\mathrm{P}<0.05)$. ${ }^{\#}$ Rounded off to three decimal. 
Table 3. eNOS haplotype in block frequencies and their associations with risk of recurrent miscarriage.

\begin{tabular}{|c|c|c|c|c|c|c|c|}
\hline \multirow[t]{2}{*}{ Block } & \multirow[t]{2}{*}{ Haplotype $^{\mathrm{a}}$} & \multirow[t]{2}{*}{ Controls (N, \%) } & \multirow[t]{2}{*}{ Cases (N, \%) } & \multicolumn{4}{|c|}{ Statistics } \\
\hline & & & & $\chi^{2}$ & $\mathrm{P}^{\mathrm{b}}$ & OR & $95 \% \mathrm{CI}$ \\
\hline \multirow[t]{3}{*}{1} & T-T-G & $120(59.701)$ & $91(47.396)$ & 5.981 & 0.015 & 0.608 & $0.408-0.907$ \\
\hline & C-T-G & $53(26.368)$ & $58(30.208)$ & 0.715 & 0.433 & 1.209 & $0.779-1.876$ \\
\hline & C-C-T & $27(13.433)$ & $31(16.146)$ & 0.575 & 0.479 & 1.241 & $0.710-2.169$ \\
\hline \multirow[t]{3}{*}{2} & A-A & $136(67.662)$ & $133(69.271)$ & 0.118 & 0.746 & 1.077 & $0.704-1.649$ \\
\hline & C-G & $50(24.876)$ & $45(23.438)$ & 0.111 & 0.814 & 0.924 & $0.582-1.468$ \\
\hline & A-G & $14(6.965)$ & $15(7.813)$ & 0.103 & 0.848 & 1.132 & $0.531-2.413$ \\
\hline \multirow[t]{3}{*}{3} & A-T & $87(43.284)$ & $81(42.188)$ & 0.048 & 0.839 & 0.956 & $0.641-1.426$ \\
\hline & C-G & $64(31.841)$ & $64(33.333)$ & 0.100 & 0.830 & 1.070 & $0.702-1.632$ \\
\hline & C-T & $49(24.378)$ & $46(23.958)$ & 0.009 & 1.000 & 0.977 & $0.616-1.551$ \\
\hline
\end{tabular}

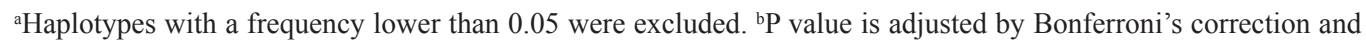
statistically significant. Results $(\mathrm{P}<0.0017$ in block $1 ; \mathrm{P}<0.025$ in blocks 2 and 3$)$.

The results revealed a significant association between the rs1799983 genotype distribution and $\mathrm{RM}$ patients (chi-square $=15.071, \mathrm{P}=0.001$ ). $\mathrm{RM}$ patients had a significantly lower frequency of the $\mathrm{G}$ allele (chi-square $=6.250, \mathrm{P}=0.016, \mathrm{OR}=1.625,95 \% \mathrm{CI}=1.108-2.383$ ). A significant between-group difference was found in the genotype distribution of rs 11771443 (chi-square $=6.259, \mathrm{P}=0.044$ ). $\mathrm{RM}$ patients had a significantly higher frequency of the $\mathrm{C}$ allele of rs11771443 (chi-square $=7.076, \mathrm{P}=0.008, \mathrm{OR}=1.497,95 \% \mathrm{CI}=1.128-1.986$ ).

Tests of the 3-marker haplotype (block 1) association using rs11771443, rs2070744, and rs 179998 provided evidence for significant associations between these SNPs and RM $(\mathrm{P}<$ 0.017). Owing to higher frequencies in healthy controls, a protective effect may be implicated with T-T-G haplotypes ( $\mathrm{P}=0.015$, after Bonferroni correction).

\section{DISCUSSION}

Recently, eNOS has been reported to be overexpressed in the glandular and luminal epithelium of the endometrium in women with RM and unexplained infertility, suggesting a detrimental effect of excess $\mathrm{NO}$ on endometrial receptivity and implantation (Najafi et al., 2012). In humans, abnormal levels of NO as well as polymorphic variants of eNOS have been shown to play important roles in pregnancy-related vascular disease (Hefler et al., 2002; Hillermann et al., 2005; Huber et al., 2005; Suryanarayana et al., 2006; Yoshimura et al., 2001).

The rs1799983 SNP alters the stability of eNOS (Tesauro et al., 2000) and is associated with reduced NO production (Veldman et al., 2002). This reduction is reportedly associated with various vascular diseases such as myocardial infarction (Shimasaki et al., 1998), placental abruption (Yoshimura et al., 2001), and pre-eclampsia (Yoshimura et al., 2000; Savvidou et al., 2001). A meta-analysis has revealed a close association between rs1799983 and idiopathic RM (Su et al., 2011). Our results demonstrated that RM patients had a significantly lower frequency of the G allele of rs1799983, which is consistent with previous studies in Koreans (Shin et al., 2010), whites (Hefler et al., 2002), and Indians (Parveen et al., 2011). By contrast, some studies have not confirmed this association (Daher et al., 2012).

Population stratification and sample size are important concerns in human genetic surveys (Thomas and Witte, 2002). In the present study, the experimental and control groups were ethnically matched by drawing from a homogeneous population. Our study had sufficient statistical power to argue an epidemiologically relevant impact of hereditary variations among 
the studied genes. The statistical difference remained significant when the data were subjected to Bonferroni correction. Furthermore, T-T-G (rs11771443-rs2070744-rs1799983) haplotypes may be involved in a protective effect. Against our expectation, the $\mathrm{G}$ allele of rs 1799983 made no other haplotype in RM patients or controls. Thus, we could safely conclude that the G allele of rs 1799983 had the greatest effect on rs11771443 and rs2070744 alleles in Chinese women.

The rs2070744 SNP in the promoter region of eNOS has been observed in patients with vascular disease (Khurana et al., 2003; Rossi et al., 2003; Guang-Da et al., 2005), and this polymorphism inhibits the promoter activity of eNOS, reducing NO production in blood vessels and causing endothelial dysfunction (Brown et al., 2003; Kimura et al., 2003; Senthil et al., 2005). Previous studies have demonstrated a lack of consistent association between the rs 2070744 polymorphism and recurrent pregnancy loss in Tunisians (Zammiti et al., 2008) and Koreans (Shin et al., 2010). The current study suggests that this polymorphism was unassociated with the development of RSA in a Chinese population sample. The most intriguing finding was the significant association between rs11771443 in the promoter of the eNOS gene and RM. To our knowledge, this study is the first to associate rs 11771443 with RM. The result suggests that this polymorphism might play a critical role in the development of RM through regulation of eNOS transcription. The functional importance of this polymorphism, however, requires further studies.

In conclusion, in a homogeneous population sample with a relatively large sample size, we found that 2 eNOS polymorphisms (rs1799983 and rs11771443) were associated with RM. These findings encourage the search for functional polymorphisms within and close to the eNOS gene using a systemic approach in a larger population sample.

\section{ACKNOWLEDGMENTS}

Research partially supported by the Shanxi Province Basic Research Development Program (\#2010k15-06-05).

\section{REFERENCES}

Athanassakis I, Aifantis I, Ranella A, Giouremou K, et al. (1999). Inhibition of nitric oxide production rescues LPSinduced fetal abortion in mice. Nitric Oxide 3: 216-224.

Ayuk PT, Theophanous D, D’Souza SW, Sibley CP, et al. (2002). L-arginine transport by the microvillous plasma membrane of the syncytiotrophoblast from human placenta in relation to nitric oxide production: effects of gestation, preeclampsia, and intrauterine growth restriction. J. Clin. Endocrinol. Metab. 87: 747-751.

Brown KS, Kluijtmans LA, Young IS, Woodside J, et al. (2003). Genetic evidence that nitric oxide modulates homocysteine: the NOS3 894TT genotype is a risk factor for hyperhomocystenemia. Arterioscler. Thromb. Vasc. Biol. 23: 1014-1020.

Daher S, Mattar R, Gueuvoghlanian-Silva BY and Torloni MR (2012). Genetic polymorphisms and recurrent spontaneous abortions: an overview of current knowledge. Am. J. Reprod. Immunol. 67: 341-347.

Guang-Da X, Qiong-shu W and Wen J (2005). A T-786C polymorphism in 5'-flanking region of the endothelial nitric oxide synthase gene and endothelium-dependent arterial dilation in Type 2 diabetes. Diabet. Med. 22: 1663-1669.

Haddad EK, Duclos AJ and Baines MG (1995). Early embryo loss is associated with local production of nitric oxide by decidual mononuclear cells. J. Exp. Med. 182: 1143-1151.

Hefler LA, Tempfer CB, Bashford MT, Unfried G, et al. (2002). Polymorphisms of the angiotensinogen gene, the endothelial nitric oxide synthase gene, and the interleukin-1beta gene promoter in women with idiopathic recurrent miscarriage. Mol. Hum. Reprod. 8: 95-100.

Hillermann R, Carelse K and Gebhardt GS (2005). The Glu298Asp variant of the endothelial nitric oxide synthase gene is associated with an increased risk for abruptio placentae in pre-eclampsia. J. Hum. Genet. 50: 415-419.

Huber A, Grimm C, Jirecek S, Heim K, et al. (2005). Polymorphisms of the Nos3 gene and unexplained late intrauterine 
fetal death. Eur. J. Obstet. Gynecol. Reprod. Biol. 122: 151-155.

Khurana VG, Sohni YR, Mangrum WI, McClelland RL, et al. (2003). Endothelial nitric oxide synthase T-786C single nucleotide polymorphism: a putative genetic marker differentiating small versus large ruptured intracranial aneurysms. Stroke 34: 2555-2559.

Kimura T, Yokoyama T, Matsumura Y, Yoshiike N, et al. (2003). NOS3 genotype-dependent correlation between blood pressure and physical activity. Hypertension 41: 355-360.

Li TC, Makris M, Tomsu M, Tuckerman E, et al. (2002). Recurrent miscarriage: aetiology, management and prognosis. Hum. Reprod. Update 8: 463-481.

Moncada S and Higgs A (1993). The L-arginine-nitric oxide pathway. N. Engl. J. Med. 329: 2002-2012.

Myatt L, Eis AL, Brockman DE, Greer IA, et al. (1997). Endothelial nitric oxide synthase in placental villous tissue from normal, pre-eclamptic and intrauterine growth restricted pregnancies. Hum. Reprod. 12: 167-172.

Najafi T, Novin MG, Ghazi R and Khorram O (2012). Altered endometrial expression of endothelial nitric oxide synthase in women with unexplained recurrent miscarriage and infertility. Reprod. Biomed. Online 25: 408-414.

Nakayama M, Yasue H, Yoshimura M, Shimasaki Y, et al. (1999). T-786 $\rightarrow$ C mutation in the 5'-flanking region of the endothelial nitric oxide synthase gene is associated with coronary spasm. Circulation 99: 2864-2870.

Parveen F, Faridi RM, Alam S and Agrawal S (2011). Genetic analysis of eNOS gene polymorphisms in association with recurrent miscarriage among North Indian women. Reprod. Biomed. Online 23: 124-131.

Rossi GP, Cesari M, Zanchetta M, Colonna S, et al. (2003). The T-786C endothelial nitric oxide synthase genotype is a novel risk factor for coronary artery disease in Caucasian patients of the GENICA study. J. Am. Coll. Cardiol. 41: 930-937.

Sanyal M, Nag TC and Das C (2000). Localization of nitric oxide synthase in human trophoblast cells: role of nitric oxide in trophoblast proliferation and differentiation. Am. J. Reprod. Immunol. 43: 70-77.

Savvidou MD, Vallance PJ, Nicolaides KH and Hingorani AD (2001). Endothelial nitric oxide synthase gene polymorphism and maternal vascular adaptation to pregnancy. Hypertension 38: 1289-1293.

Senthil D, Raveendran M, Shen YH, Utama B, et al. (2005). Genotype-dependent expression of endothelial nitric oxide synthase (eNOS) and its regulatory proteins in cultured endothelial cells. DNA Cell Biol. 24: 218-224.

Serrano NC, Casas JP, Diaz LA, Paez C, et al. (2004). Endothelial NO synthase genotype and risk of preeclampsia: a multicenter case-control study. Hypertension 44: 702-707.

Shimasaki Y, Yasue H, Yoshimura M, Nakayama M, et al. (1998). Association of the missense Glu298Asp variant of the endothelial nitric oxide synthase gene with myocardial infarction. J. Am. Coll. Cardiol. 31: 1506-1510.

Shin SJ, Lee HH, Cha SH, Kim JH, et al. (2010). Endothelial nitric oxide synthase gene polymorphisms (-786T $>$ C, 4a4b, $894 \mathrm{G}>\mathrm{T}$ ) and haplotypes in Korean patients with recurrent spontaneous abortion. Eur. J. Obstet. Gynecol. Reprod. Biol. 152: 64-67.

Su MT, Lin SH and Chen YC (2011). Genetic association studies of angiogenesis- and vasoconstriction-related genes in women with recurrent pregnancy loss: a systematic review and meta-analysis. Hum. Reprod. Update 17: 803-812.

Suryanarayana V, Rao L, Kanakavalli M, Padmalatha V, et al. (2006). Recurrent early pregnancy loss and endothelial nitric oxide synthase gene polymorphisms. Arch. Gynecol. Obstet. 274: 119-124.

Tesauro M, Thompson WC, Rogliani P, Qi L, et al. (2000). Intracellular processing of endothelial nitric oxide synthase isoforms associated with differences in severity of cardiopulmonary diseases: cleavage of proteins with aspartate vs. glutamate at position 298. Proc. Natl. Acad. Sci. U. S. A. 97: 2832-2835.

Thomas DC and Witte JS (2002). Point: population stratification: a problem for case-control studies of candidate-gene associations? Cancer Epidemiol. Biomarkers Prev. 11: 505-512.

Veldman BA, Spiering W, Doevendans PA, Vervoort G, et al. (2002). The Glu298Asp polymorphism of the NOS 3 gene as a determinant of the baseline production of nitric oxide. J. Hypertens. 20: 2023-2027.

Yamada H, Kato EH, Kobashi G, Ebina Y, et al. (2001). Recurrent pregnancy loss: etiology of thrombophilia. Semin. Thromb. Hemost. 27: 121-129.

Yoshimura T, Yoshimura M, Tabata A, Shimasaki Y, et al. (2000). Association of the missense Glu298Asp variant of the endothelial nitric oxide synthase gene with severe preeclampsia. J. Soc. Gynecol. Investig. 7: 238-241.

Yoshimura T, Yoshimura M, Tabata A, Yasue H, et al. (2001). The missense Glu298Asp variant of the endothelial nitric oxide synthase gene is strongly associated with placental abruption. Hum. Genet. 108: 181-183.

Zammiti W, Mtiraoui N and Mahjoub T (2008). Lack of consistent association between endothelial nitric oxide synthase gene polymorphisms, homocysteine levels and recurrent pregnancy loss in tunisian women. Am. J. Reprod. Immunol. 59: $139-145$.

Zygmunt M, Herr F, Keller-Schoenwetter S, Kunzi-Rapp K, et al. (2002). Characterization of human chorionic gonadotropin as a novel angiogenic factor. J. Clin. Endocrinol. Metab. 87: 5290-5296. 